\title{
Evaluación de Paratheresia claripalpis y Metagonistylum minense como posibles parasitoides de larvas de Rhynchophorus palmarum, bajo condiciones de laboratorio en el municipio de Buenaventura, Valle del Cauca, Colombia
}

\section{Evaluation of Paratheresia claripalpis and Metagonistylum minense you as possible parasitoides of larvas of Rhynchophorus palmarum, under conditions of laboratory in the municipality of Buenaventura, Valle del Cauca, Colombia}

\author{
Moisés Mosquera Blandón ${ }^{1}$, Jesús David Viáfara ${ }^{2}$ \\ RESUMEN
}

El anillo rojo es la principal enfermedad que afecta las plantaciones de cocotero (Cocos nucifera) en el litoral Pacífico colombiano, ocasionando graves pérdidas económicas a los agricultores, porque es una enfermedad de carácter letal. Su agente causal es el nemátodo Bursaphelenchus coccophilus y su vector el insecto Rhynchophorus palmarum, los cuales actúan en intima asociación. Con el objetivo de generar información que condujera a establecer recomendaciones adecuadas para el manejo de esta enfermedad, el IIAP adelantó un proceso de investigación concluyendo que la enfermedad está presente en las plantaciones de cocotero variedad típico, ubicadas en la bocana de Guapi (estero La Preba), vereda Chanzará, Quiroga (estero pejesapo) y en el municipio de Timbiquí (estero pampanilla) Cuerval. Este estudio también demostró que Paratheresia claripalpis y Metagonistylum minense, no son parásitos potenciales de larvas de R. palmarum bajo condiciones de laboratorio, porque después del tiempo estipulado (12 días), no se presentó la muerte de ninguna de las larvas, por efecto de la parasitación de los tachinidae.

Palabras clave: Enfermedad anillo rojo; Bursaphelenchus coccophilus; Cocos nucifera; Metagonistylum minense; Paratheresia claripalpis; Rhynchophorus palmaru.

\begin{abstract}
The red ring is the main illness that affects the coconut tree plantations (Coconuts nucifera) in the coast Colombian Pacific, causing serious economic losses to the farmers, being an illness of lethal character. The causal agent of the illness is the nemátodo Bursaphelenchus coccophilus and her vector is the insect Rhynchophorus palmarum, which act in he/she becomes intimate association. With the objective of generating information that drove to establish appropriate recommendations for the handling of this illness, the IIAP advanced an investigation process concluding that the illness is present in the plantations of typical coconut tree variety, located in the bocana of Guapi (estero la Preba), sidewalk of Chanzará, Quiroga (estero pejesapo) and in the municipality of Timbiqui (estero pampanilla) Cuerval. This study also demonstrated that Paratheresia claripalpis and Metagonistylum mine you, is not potential parasites of larvas of R. palmarum low laboratory conditions, since after the specified time (12 days), you doesn 't present the death of none of the larvas, for effect of the parasitación of the tachinidae.
\end{abstract}

1. Investigador principal, Componente Productivo, Instituto de Investigaciones Ambientales del Pacífico(IIAP), Quibdó, Colombia.

e-mail:moisesmosquera11@gmail.com

2. Estudiante de agronomía, Universidad del Pacífico, Buenaventura, Colombia. e-mail: viafarajdv@gmail.com

Recibido: agosto 19, 2008 Aceptado: septiembre 2, 2008 
Keywords: Illness red ring; Bursaphelenchus coccophilus; Cocos nucifera; Metagonistylum minense; Paratheresia claripalpis; Rhynchophorus palmaru.

\section{INTRODUCCIÓN}

En procura de generar información que condujera a establecer recomendaciones adecuadas para el manejo de la enfermedad del cocotero, anillo rojo, en territorios colectivos de comunidades negras de la costa pacífica colombiana, el Instituto de Investigaciones Ambientales del Pacífico (IIAP), Quibdó, Colombia, adelantó un proceso de investigación (tesis de grado) con recursos económicos del presupuesto nacional, tomando como sitios de muestreo 11 fincas ubicadas en el estero pejesapo vereda Quiroga, estero la preba, quebrada Los Mellizos y estero el bagrero bocana de Guapi y en el estero pampanilla, Cuerval, municipio de Timbiquí.

El anillo rojo es la principal enfermedad que afecta las plantaciones de cocotero (Cocos nucifera) en el litoral Pacífico colombiano, ocasionando graves pérdidas económicas a los agricultores, porque la enfermedad es de carácter letal. El agente causal es el nemátodo Bursaphelenchus coccophilus y su vector el insecto Rhynchophorus palmarum, los cuales actúan en intima asociación. Este insecto en estado larval ocasiona daños mecánicos graves (galerías), tanto en palmas sanas como enfermas. Además, en estado adulto el insecto lleva en su cuerpo nemátodos, que son inoculados al visitar palmas sanas, cobrando así mayor poder destructivo. Por ello, se plantea que el manejo de la enfermedad debe, necesariamente, involucrar la reducción de los niveles de población del insecto.

El objetivo principal de este estudio es evaluar bajo condiciones de laboratorio a Paratheresia claripalpis y Metagonistylum minense como posibles parasitoides de larvas de Rhynchophorus palmarum en el municipio de Buenaventura.

\section{AREADEESTUDIO}

El área de estudio se encuentra ubicada a los $2^{\circ} 34^{\prime}$ de latitud norte y a los $75^{\circ} 54^{\prime}$ de longitud occidental del suroccidente del departamento del Cauca, Colombia. En esta zona predominan las condiciones de bosque muy húmedo tropical (bmh-T), presenta una temperatura media anual de $26^{\circ} \mathrm{C}$, altitud $5 \mathrm{msnm}$, precipitación media anual que fluctúa entre 4.000 y $8.000 \mathrm{~mm}$, humedad relativa de $92 \%$ y 3 horas día de brillo solar.

La localización estratégica y condiciones agroambientales de este sector, influye en aspectos como su biodiversidad y suelos con alto grado de vocación para el establecimiento de esta especie, situación que ha generado el establecimiento de cultivos comerciales del cocotero y se constituya en una

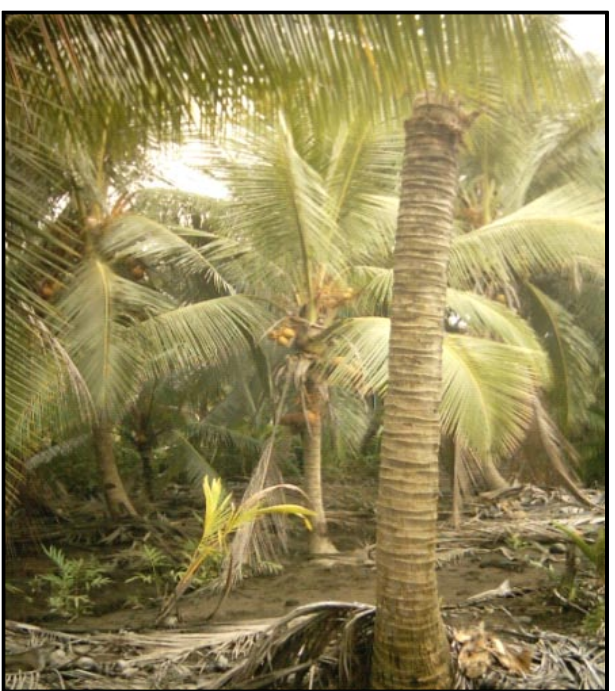

\section{Figura 1. Palma de coco (Cocos nucifera) en su fase terminal afectada por la enfermedad anillo rojo}

fuente importante de ingresos y mano de obra para la población local. Sin embargo, factores como el desconocimiento del manejo fitosanitario de las plantaciones por parte de los nativos y la realización de actividades productivas sin criterios técnicos adecuados para la conservación y protección del medio natural, ha generado la pérdida de grandes extensiones de áreas cultivadas y acentuado una profunda crisis socioeconómica y ambiental en esta región del Chocó Biogeográfico.

Situaciones como las descritas, hacen necesario la toma de medidas por parte de institutos de investigaciones y autoridades ambientales, de tal manera que se detenga el avance de esta letal enfermedad y se garantice la sostenibilidad económica y ambiental de esta actividad productiva. Por ello, se hace absolutamente necesario el conocimiento profundo de la inter-relación entre el organismo vector y sus enemigos naturales para, a través de ello, mantener un control de la población con alternativas diferentes al control químico, que afecta el equilibrio natural en estos importantes ecosistemas costeros del Pacífico colombiano.

\section{MÉTODOS}

Con el fin de conocer el índice de afección y estado de las plantaciones en la zona, en la fase de campo se realizaron 11 visitas a fincas de cocotero, ubicadas en el estero pejesapo vereda Quiroga, estero la preba, quebrada Los Mellizos y estero el bagrero bocana de Guapi y en el estero pampanilla, Cuerval, municipio de Timbiquí. Las larvas Rhynchophorus palmarum se colectaron en 3 plantaciones de coco (Cocos nucifera) sembradas con variedad típico, infectadas por la enfermedad anillo rojo (Figura 1). La primera plantación tiene 


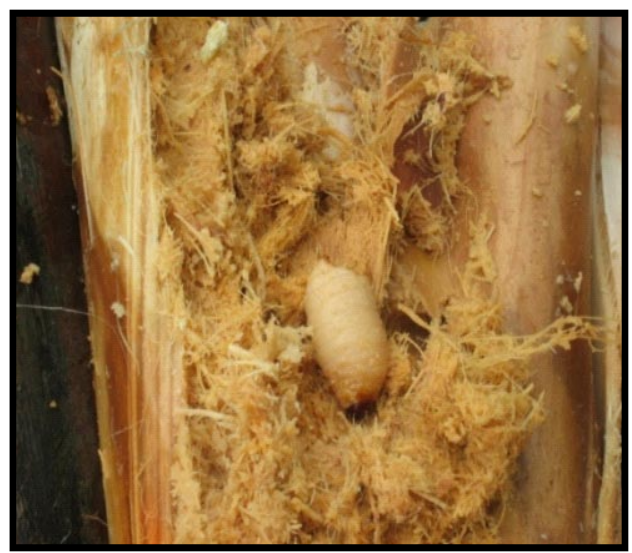

Figura 2. Larva de Rhynchophorus palmarumencontrada en el cogollo de la palma tumbada

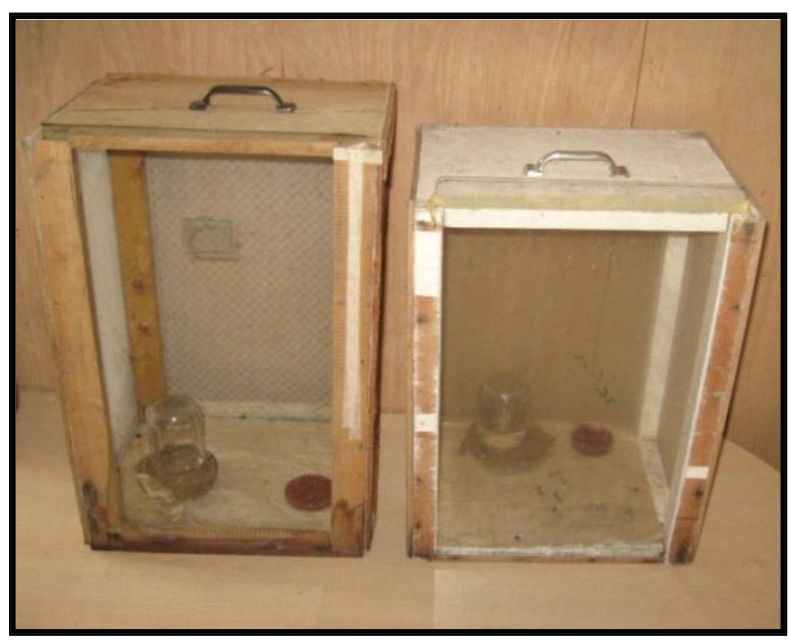

Figura 4. Jaulas de emergencia de los parasitoides M. minensey P. claripalpis

unárea de 5 hectáreas, la segunda 20 y la tercera 25. Todas con 30 años de establecimiento, ubicada en el estero la preba bocana del municipio de Guapi.

En forma aleatoria se seleccionaron las palmas enfermas de anillo rojo, para luego tumbarlas. Una vezubicadas, se hizo un corte trasversal a una altura entre $50-70 \mathrm{~cm}$ del suelo. Enseguida se realizaron cortes en el cogollo, tronco de la palma y estipe de las hojas, que dejaron en evidencia las galerías, producto de la acción fagocitaria de las larvas de $R$. palmarum, lo que permitió su recolección.

Las larvas recolectadas en campo (Figura 2), se depositaron en una caja de madera, que contenían trozos de cogollo y estipite de la hoja de la palma para su alimentación y permitirles un ambiente adecuado de humedad y temperatura. Luego se transportaron hasta el municipio de Guapi, donde se introdujeron en cajas con malla con compartimientos internos (Figura 3), por efecto del canibalismo existente entre

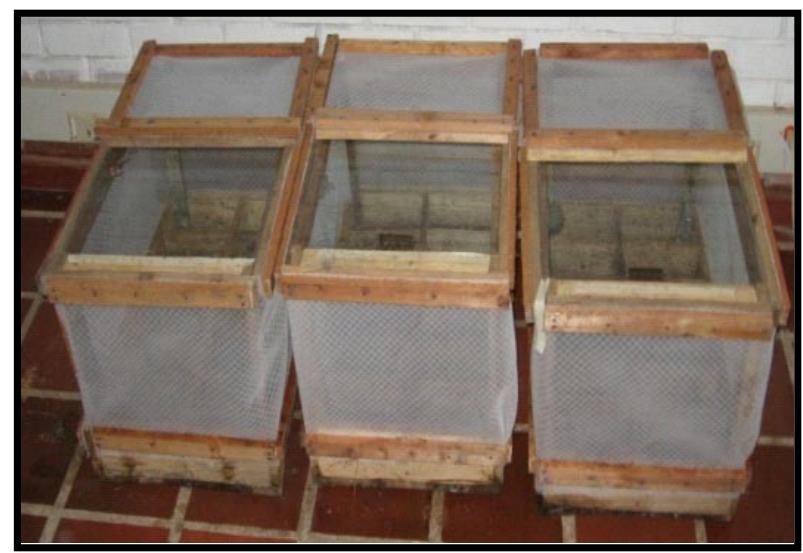

Figura 3. Cajas con compartimientos para colocar las larvas de $R$. palmarum

ellas. El sustrato que contenían estas cajas se esterilizó previamente utilizando 6 litros de agua caliente $\left(90^{\circ} \mathrm{C}\right)$ durante 5 minutos. Después se dejó enfriar durante 45 minutos a temperatura ambiente. Las larvas recolectadas se trasladaron al laboratorio de la Universidad del Pacífico ubicada en Buenaventura, Colombia. Una vez las larvas se encontraban en el laboratorio con una temperatura $28^{\circ} \mathrm{C}$ y humedad relativa de $82 \%$, se dejaron 6 larvas por caja. En dichas cajas se colocaron $2 \mathrm{~kg}$ de trozos de palma de coco, $200 \mathrm{~g}$ en cada compartimiento. Las larvas se mantuvieron ahí, hasta que los parasitoides estuvieran listos para ser inoculados. Estos parasitoides se obtuvieron en estado de pupa, en los laboratorios de insumos biológicos ubicados en la ciudad de Palmira (Bioecológicos para P. claripalpis) y en la ciudad de Buga (Probiol para M. minense). Se colocó cada especie en una jaula hasta que emergieron los adultos (Figura 4). Ambos tuvieron una emergencia del $100 \%$. Luego, se introdujo en las jaulas agua y mermelada de guayaba para su alimentación. Para alcanzar la madurez de $M$. minense se dejó durante 9 días y para $P$. claripalpis 13 días. Cuando los adultos alcanzaron su madurez (10 días), estos se llevaron a las cajas con compartimiento. Se utilizaron 7 cajas, una se dejó como testigo con 6 larvas de $R$. palmarum sin parasitoides. Las 6 restantes, contenían 6 larvas de $R$. palmarum por caja. En 3 de estas se colocaron 3 hembras adultas por caja de $M$. minense y 3 de P. claripalpis) (Figura 5). Teniendo así 2 tratamientos con 3 repeticiones y un testigo; cada caja fue la unidad experimental y se distribuyó en un diseño completamente aleatorio.

Luego las cajas se taparon con papel kraft, proporcionando penumbra. Las cajas con los parasitoides se dejaron durante 12 días. En todo este tiempo se revisó diariamente para observar el comportamiento de las larvas y luego realizar las evaluaciones del caso. Además, se roseaba agua para humedecer el sustrato donde estaban las larvas de $R$. palmarum. 


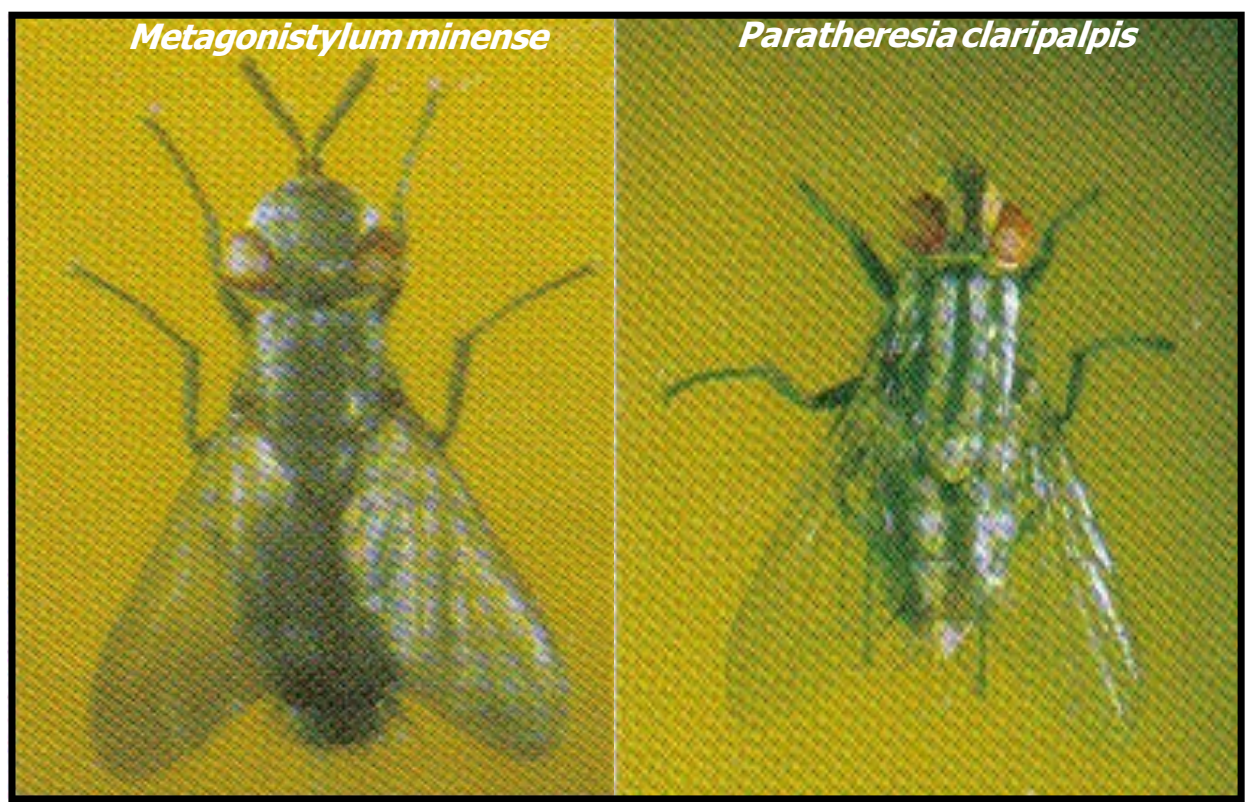

Figura 5. Adultos de Tachinidae utilizados en la prueba de parasitismo

En el laboratorio Bioecológicos, se realizó la inoculación directa tomando hembras de $P$. claripalpis y $M$. minense con larvitas o maggots maduras en el ovisaco; es decir, hembras fertilizadas entre los 8 y 12 días después de la cópula. Estas hembras fueron disceptadas y se extrajo con mucho cuidado el ovisaco, colocándolos sobre una cápsula de 3 a $5 \mathrm{~cm}$ de diámetro de color negro. Previamente, los adultos se desinfectaron con solución suave de hipoclorito al $2 \%$. Luego se agregó sobre la cápsula una gota de suero fisiológico para evitar la desecación; seguidamente, se perforaron los ovisaco para permitir la salida gradual de los parásitos o maggots.

Con un pincel fino $\mathrm{N}^{\circ} 4$ se levantaron 10 parásitos (maggots) y se colocaron en la región dorsal de las larvas de $R$. palmarum de 7 a 9 instar. Se parasitaron 10 larvas de R. palmarum con $P$. claripalpis y 10 larvas con $M$. minense. Por último, las larvas parasitadas se dejaron por separado en un frasco. Estos frascos se guardaron en un lugar oscuro y se hicieron revisiones diarias.

\section{RESULTADOSYDISCUSIÓN}

Los resultados obtenidos en este estudio, indican que Paratheresia claripalpis y Metagonistylum minense, no son parásitos potenciales de larvas de R. palmarum bajo condiciones de laboratorio, porque después del tiempo estipulado (12 días), no se presentó la muerte de ninguna de las larvas, por efecto de la parasitación de los Tachinidae, pues no se encontraron larvas ni pupas de los parasitoides en las larvas del insecto. Se deduce que no hubo parasitación. Las larvas que se encontraron muertas (Tabla 1), fue por causas externas, posiblemente a la temperatura.
Sin embargo, Moura etal. (1993), informan que en evaluaciones realizadas en plantaciones de palma aceitera (Elaeis guineensis) en el sur del estado de Bahía, Brasil, atacadas por R. palmarum, se determinó un parasitismo promedio de $51 \%$, con un promedio de 18.3 pupas de Paratheresia menezesi encontradas en los picudos parasitados. Además, se informa $37 \%$ de parasitismo de Amerrhynus ynca por Paratheresia menezesi bajo condiciones de campo. Esta es una de las principales plagas del cocotero en el sur de Bahía, Brasil (Moura etal., 2002).

Igualmente, Moura et al., (2006), describen que en condiciones de campo se encontró Billaea rhynchophoreae (Diptera: Tachinidae), parasitando Rhynchophorus palmarum (Coleoptero: Curculionidae), donde la media de parasitación fue $40 \%$.

Debido a la respuesta negativa del parasitismo de los Tachinidae sobre larvas de R. palmarum, utilizando la técnica de adultos, se realizó la técnica de inoculación con larvas. Infortunadamente por problemas de roedores, no fue posible culminar con éxito este ensayo. Por cuestión de tiempo y dinero este experimento no se logró repetir. Sin embargo, los parásitos o maggots de Paratheresia claripalpis o Metagonistylum minense cuando se inoculan sobre los pliegues dorsales de Diatraea saccharalis (Lepidoptero: Pyralidae), a los 8 ó 10 días destruyen totalmente las partes internas de la larva (Costilla et al., 1991).

Las inoculaciones hechas bajo condiciones de laboratorio en Diatraea spp, utilizando a Paratheresia claripalpis y Metagonistylum minense, en 2 a 3 minutos penetran por los pliegues dorsales de su huésped. A los 4 días, las larvas disminuyen su actividad de alimentación y a 11 días después 
Bioetnia Volumen 5 № 2 (julio-diciembre), 2008

Tabla 1

Números de larvas vivas y muertas de $R$ hynchophorus palmarum, después de ser sometidas a parasitación con Paratheresia claripalpisy Metagonistylum minense

\begin{tabular}{lcc}
\hline Paratheresia claripalpis & 6 & 12 \\
Metagonistylum minense & 7 & 11 \\
Testigo & 4 & 2 \\
\hline
\end{tabular}

de la inoculación emergen los puparios de los parásitos.

El desarrollo de esta investigación y sus resultados permiten concluir que la enfermedad del anillo rojo está presente en las plantaciones de cocotero variedad típico, ubicadas en la bocana de Guapi (estero la preba), vereda de Chanzará, Quiroga (estero pejesapo) y en el municipio de Timbiquí (estero pampanilla) Cuerval, disminuyendo la producción y ocasionando grandes pérdidas económicas en los agricultores. El transporte vía marítima afecta de forma significativa el período de vida de las larvas de Rhynchophorus palmarum. Los parasitoides Paratheresia claripalpis y Metagonistylum minense no son parásitos potenciales de larvas de Rhynchophorus palmarum bajo condiciones de laboratorio.

\section{RECOMENDACIONES}

Se recomienda realizar la recolección de larvas de Rhynchophorus palmarum en campo, tener mucho cuidado al hacer los cortes transversales y longitudinales en busca de las galerías donde se encuentran las larvas, porque el contacto del machete o hacha con la larva le puede ocasionar la muerte.

- La recolección de larvas de R. palmarum debe ser continua en plantaciones que manifiesten la enfermedad del anillo rojo.

- La conservación de las larvas de Rhynchophorus palmarum con fines investigativos, debe ser en un recipiente que conserve humedad y temperatura adecuada y contenga compartimiento, porque existe canibalismo entre ellas.

- Realizar ensayos como este, aumentando la dosis de parasitoides a 6 hembras o más por unidad experimental.

- Realizar ensayos bajo condiciones de laboratorio, haciendo inoculación directa de los parásitos Paratheresia claripalpis y Metagonistylum minense sobre las larvas de Rhynchophorus palmarum.

Realizar investigaciones sobre la búsqueda de enemigos naturales en campo del insecto Rhynchophorus palmarum. Estudiar Tachinidae bajo condiciones de laboratorio como
Paratheresia menezesi y Billaea rhynchophorae son posibles agentes controladores de $R$. palmarum.

\section{LITERATURA CITADA}

Alianza PBS del Pacífico (Promotora de Bienes y Servicios del Pacífico), Aso Manos Negra y la Asociación de Consejos Comunitarios de Timbiquí. 2006. El programa piloto de desarrollo agroindustrial del coco y mejoramiento de las condiciones de vida de la población afro-colombiana vulnerable de la costa caucana. Chocó: Alianza PBS del Pacífico (Promotora de Bienes y Servicios del Pacífico), Aso Manos Negra y la Asociación de Consejos Comunitarios de Timbiquí; p. 4-7.

Carrasquilla, M. A. 2006. Situación actual y manejo de problemas sanitarios del cultivo de cocotero (Cocos nucifera) en la costa nariñense, Tumaco, Nariño. p. 1-7.

Corporación Autónoma Regional del Cauca (CRC), Plan de Ordenamiento Territorial (POT). Disponible en: http://www.crc.gov.co/pot/ potguapi.htm. [fecha de acceso abril, 2008]

CORPOICA. 1999. Manejo del picudo (Rhynchophorus palmarum) insecto vector del nemátodo causante de la enfermedad anillo rojo del cocotero (Cocos nucifera). Tumaco: Programa Nacional de Transferencia de Tecnología (PRONNATA). p. 5-8.

Costilla, A. M., Osores M. V., Willink, E. 1991. Biología de la mosca Paratheresia claripalpis wulp y su cría en laboratorio. Rev Ind Agricola de Tucumán. 68 (1-2): 219-30.

García, T. O. 1986. Radinaphelenchus cocophilus asociado al anillo rojo y a la enfermedad de la hoja pequeña en el cocotero. Habana: Editorial REILLY CIDA; p. 7.

Mexzón G, R., Chinchilla M, C., Castillo, G., Salamanca, D. 1994. Biología y hábito de Rhynchophorus palmarum $L$. asociado a la palma aceitera en Costa Rica. Danny. ASD Oil Palm. 8; 14-21.

Moura, J. I. L, Mauriau, D., Delabie, J.H.C. 1993. Eficiência de Paratheresia menezesi Townsend (Diptera: Tachidae) no controle biológico natural de Rhynchophorus palmarum (L.) (Coleóptera: Curculionidae). Oléagineux. 48 (5): 219-23.

Moura, L. J. I., Fanton, C. J., Mesquita, B. C. A. 2002. Evaluación de un método agronómico, biológico y químico para el combate de Amerrhinus ynca en plantaciones de coco. Manejo Integrado de Plagas y Agroecolgía (Costa Rica). 65: 109-12.

Moura, L. J. I, Toma, R., Sgrillo B. R., Delabie H. C. J. 2006. Natural efficiency of parasitism by Billaea rhynchophorae (Blanchard) (Diptera: Tachinidae) for the control of Rhynchophorus palmarum (L) (Coleoptera: Curculionidae). Neotropical Entomol. 35 (2): 273-4.

Peña, R. E. A. 1995a. La enfermedad anillo rojo: Etiología y sintomatología. En: Memorias del seminario de actualización de conocimientos sobre el cultivo del cocotero (Cocos nucifera). Guapi: Instituto Colombiano Agropecuario (ICA) subregional de prevención y control; p. 7.

Vélez, R. A. 1997. Plagas agrícolas de impacto económico en Colombia: bionomía y manejo integrado. $2^{\mathrm{a}}$ ed. Medellín: Editorial Universidad de Antioquia; p. 150-6. 